\title{
On the existence of $L^{2}$-valued thermodynamic entropy solutions for a hyperbolic system with boundary conditions
}

\section{Stefano Marchesani \& Stefano Olla}

To cite this article: Stefano Marchesani \& Stefano Olla (2020) On the existence of $L^{2}$-valued thermodynamic entropy solutions for a hyperbolic system with boundary conditions, Communications in Partial Differential Equations, 45:9, 1072-1087, DOI: 10.1080/03605302.2020.1750426

To link to this article: https://doi.org/10.1080/03605302.2020.1750426

册 Published online: 22 Apr 2020.

Submit your article to this journal $\pi$

山 Article views: 68

Q View related articles ¿

View Crossmark data $\nearrow$

Citing articles: 1 View citing articles 


\title{
On the existence of $L^{2}$-valued thermodynamic entropy solutions for a hyperbolic system with boundary conditions
}

\author{
Stefano Marchesani ${ }^{\mathrm{a}}$ (D) and Stefano Olla ${ }^{\mathrm{b}}$ (D) \\ ${ }^{a}$ GSSI, L'Aquila, Italy; ${ }^{b}$ CEREMADE, UMR-CNRS, Université de Paris Dauphine, PSL Research University, \\ Paris, France
}

\begin{abstract}
We prove existence of $L^{2}$-weak solutions of a quasilinear wave equation with boundary conditions. This describes the isothermal evolution of a one dimensional non-linear elastic material, attached to a fixed point on one side and subject to a force (tension) applied to the other side. The $L^{2}$-valued solutions appear naturally when studying the hydrodynamic limit from a microscopic dynamics of a chain of anharmonic springs connected to a thermal bath. The proof of the existence is done using a vanishing viscosity approximation with extra Neumann boundary conditions added. In this setting we obtain a uniform a priori estimate in $L^{2}$, allowing us to use $L^{2}$ Young measures, together with the classical tools of compensated compactness. We then prove that the viscous solutions converge to weak solutions of the quasilinear wave equation strongly in $L^{p}$, for any $p \in[1,2)$, that satisfy, in a weak sense, the boundary conditions. Furthermore, these solutions satisfy, beside the local Lax entropy condition, the Clausius inequality: the change of the free energy is bounded by the work done by the boundary tension. In this sense they are the correct thermodynamic solutions, and we conjecture their uniqueness.
\end{abstract}

\section{ARTICLE HISTORY}

Received 8 February 2019

Accepted 17 January 2020

\section{KEYWORDS}

Hyperbolic conservation laws; quasi-linear wave equation; boundary conditions; weak solutions; vanishing viscosity; compensated compactness; entropy solutions;

Clausius inequality

\section{MATHEMATICS SUBJECT} CLASSIFICATION

35L40; 35D40

\section{Introduction}

The problem of existence of weak solutions for hyperbolic systems of conservation law in a bounded domain has been studied for solutions that are of bounded variation or in $L^{\infty}$ [1]. In the scalar case some works extend to $L^{\infty}$ solutions, obtained from viscous approximations [2]. But viscous approximations require extra boundary conditions that are usually taken of Dirichlet type.

We present here an approach based on viscosity approximations, where the extra boundary conditions are of Neumann type, to reflect the conservative nature of the viscous approximation. We consider here the quasilinear wave equation

$$
\left\{\begin{array}{l}
r_{t}-p_{x}=0 \\
p_{t}-\tau(r)_{x}=0
\end{array}, \quad(t, x) \in \mathbb{R}_{+} \times[0,1]\right.
$$

where $\tau(r)$ is a strictly increasing regular function of $r$ such that $0<c_{1} \leq \tau^{\prime}(r) \leq c_{2}$, for some constant $c_{1}, c_{2}$. In Section 2, we will require some more technical assumption 
for $\tau$. We add to the system the following boundary conditions:

$$
p(t, 0)=0, \quad \tau(r(t, 1))=\bar{\tau}(t)
$$

and initial data

$$
r(0, x)=r_{0}(x), \quad p(0, x)=p_{0}(x) .
$$

The boundary tension $\bar{\tau}: \mathbb{R}_{+} \rightarrow \mathbb{R}$ is smooth and bounded with bounded derivative.

Eq. (1.1) describes the isothermal evolution of an elastic material in Lagrangian coordinates. The material point $x \in[0,1]$ has a volume strain $r(t, x)$ at time $t$ (that can also have negative values), and momentum (velocity) $p(t, x)$. The Eulerian position of the material point $x$, with respect to the position of the particle 0 , is given by $q(t, x)=\int_{0}^{x} r(t, y) d y$, so that we can identify the position of the material point $x=1$ as the total extension of the material:

$$
L(t)=q(t, 1)=\int_{0}^{1} r(t, y) d y .
$$

Let $T<\infty$ be given and arbitrary, and define $Q_{T}:=[0, T] \times[0,1]$. We shall construct weak solutions $\bar{u}(t, y)=(\bar{r}(t, y), \bar{p}(t, y)),(t, y) \in Q_{T}$, to the quasilinear wave equation such that $\bar{u}(t, \cdot) \in L^{2}(0,1)$ for all $t \leq T$ and satisfy the initial and boundary conditions in the following weak sense:

$$
\begin{gathered}
\int_{0}^{1} \varphi(t, x) \bar{r}(t, x) d x-\int_{0}^{1} \varphi(0, x) r_{0}(x) d x=\int_{0}^{t} \int_{0}^{1}\left(\varphi_{s} \bar{r}-\varphi_{x} \bar{p}\right) d x d s \\
\int_{0}^{1} \psi(t, x) \bar{p}(t, x) d x-\int_{0}^{1} \psi(0, x) p_{0}(x) d x=\int_{0}^{t} \int_{0}^{1}\left(\psi_{s} \bar{p}-\psi_{x} \tau(\bar{r})\right) d x d s+\int_{0}^{t} \psi(s, 1) \bar{\tau}(s) d s
\end{gathered}
$$

for all functions $\varphi, \psi \in C^{1}\left(Q_{T}\right)$ such that $\varphi(t, 1)=\psi(t, 0)=0$ for all $t \geq 0$.

Define the free energy of the system, associated to a profile $u(x)=(r(x), p(x)) \in$ $L^{2}(0,1)$, as

$$
\mathcal{F}(u):=\int_{0}^{1}\left(\frac{p^{2}(x)}{2}+F(r(x))\right) d x
$$

where $F(r)$ is a primitive of $\tau(r)\left(F^{\prime}(r)=\tau(r)\right)$, such that $\frac{c_{1}}{2} r^{2} \leq F(r) \leq \frac{c_{2}}{2} r^{2}$ for any $r \in$ $\mathbb{R}$. This is possible thanks to the bounds we required on $\tau^{\prime}$.

The solution $\bar{u}$ of (1.5) and (1.6) that we obtain has the following properties:

- $\bar{u} \in L^{\infty}\left(0, T ; L^{2}(0,1)\right)$

- $\bar{u}(0, x)=u_{0}(x)$ for a.e. $x$;

- For any $\phi \in C^{1}([0,1])$, the application

$$
t \mapsto \int_{0}^{1} \phi(x) \bar{u}(t, x) d x
$$

is Lipschitz continuous over $[0, T]$;

- $\quad \bar{u}$ satisfies Clausius inequality:

$$
\mathcal{F}(\bar{u}(t))-\mathcal{F}\left(u_{0}\right) \leq W(t), \quad \forall t \in[0, T]
$$


where $u_{0}=\left(r_{0}, p_{0}\right)$ and

$$
W(t):=-\int_{0}^{t} \bar{\tau}^{\prime}(s) \int_{0}^{1} \bar{r}(s, x) d x d s+\bar{\tau}(t) \int_{0}^{1} \bar{r}(t, x) d x-\bar{\tau}(0) \int_{0}^{1} r_{0}(x) d x
$$

is the work done by the external tension up to time $t$. In this sense, we call our solution a thermodynamic entropy solution. For general discussion of the connection of such thermodynamic solutions to the usual definition of entropic solutions, see [3,4].

Remark. If $\bar{r}(t, x)$ is differentiable with respect to time, we may perform an integration by parts and obtain

$$
W(t)=\int_{0}^{t} \bar{\tau}(s) d L(s)
$$

This recovers the usual mechanical definition of the work.

The construction of the solution is obtained from the following viscosity approximation

$$
\left\{\begin{array}{l}
r_{t}^{\delta}-p_{x}^{\delta}=\delta r_{x x}^{\delta} \\
p_{t}^{\delta}-\tau\left(r^{\delta}\right)_{x}=\delta p_{x x}^{\delta}
\end{array}, \quad(t, x) \in \mathbb{R}_{+} \times[0,1]\right.
$$

with boundary conditions

$$
p^{\delta}(t, 0)=0, \quad \tau\left(r^{\delta}(t, 1)\right)=\bar{\tau}(t), \quad p_{x}^{\delta}(t, 1)=0, \quad r_{x}^{\delta}(t, 0)=0
$$

and initial data

$$
r^{\delta}(0, x)=r_{0}^{\delta}(x), \quad p^{\delta}(0, x)=p_{0}^{\delta}(x)
$$

such that $r_{0}^{\delta}$ and $p_{0}^{\delta}$ are compatible with the boundary conditions, regular enough (see (3.4) and (3.5)) and converge to $r_{0}$ and $p_{0}$, respectively, as $\delta \rightarrow 0$.

Note that in the viscous approximation we have added two Neumann boundary conditions that reflect the conservative nature of the viscous perturbation. Under these conditions we have

$$
\int_{0}^{1}\left|u^{\delta}(t, x)\right|^{2} d x+\delta \int_{0}^{t} \int_{0}^{1}\left|u_{x}^{\delta}(s, x)\right|^{2} d x d s \leq C, \quad \forall t \geq 0
$$

where $C$ is independent of $t$ and $\delta$. It is thus clear that $\left\{u^{\delta}\right\}_{\delta>0}$ and $\left\{\sqrt{\delta} u_{x}^{\delta}\right\}_{\delta>0}$ are uniformly bounded in $L^{2}\left(Q_{T}\right)$. Then we rely on the existence of a family of bounded Lax entropy-entropy fluxes as in [5-7], that allows us to apply the compensated compactness in the $L^{2}$ version. The conditions assumed on $\tau(r)$ are in fact those required to apply [7] results. Under a slight different set of conditions, another $L^{p}$ extension of the compensated compactness argument can be found in [8].

\subsection{Physical motivations}

The problem arises naturally considering hydrodynamic limit for a non-linear chain of anharmonic oscillators in contact with a heat bath at a given temperature $[9,10]$. This microscopic dynamics models an isothermal transformation with two locally conserved quantities that evolve, on the macroscopic scale, following (1.1). 
Consider $N+1$ particles on the real line and, for $i=0, \ldots N$, call $q_{i}$ and $p_{i}$ the positions and the momenta of the $i$ th particle, respectively. Particles $i$ and $i-1$ interact via a nonlinear potential $V\left(q_{i}-q_{i-1}\right)$. Particle $i=0$ is at position $q_{0}=0$ and does not move, i.e. $p_{0}(t)=0$. There is a time dependent force (tension) $\bar{\tau}(t)$ acting on the last particle. Then, defining $r_{i}:=q_{i}-q_{i-1}$ we have a system with Hamiltonian

$$
H_{N}(t)=\sum_{i=1}^{N}\left(\frac{p_{i}^{2}}{2}+V\left(r_{i}\right)\right)-\bar{\tau}(t) \sum_{i=1}^{N} r_{i} .
$$

The interaction with a heat bath at temperature $\beta^{-1}$ is modeled by a stochastic perturbation of the dynamics that acts as a microscopic stochastic viscosity. Defining the discrete gradient and laplacian as

$$
\nabla a_{i}=a_{i+1}-a_{i}, \quad \Delta a_{i}=a_{i+1}+a_{i-1}-2 a_{i},
$$

the evolution equations are then given by the following system of stochastic differential equations:

$$
\begin{cases}d r_{1}=p_{1} d t+\delta \nabla V^{\prime}\left(r_{1}\right) d t-\sqrt{2 \beta^{-1} \delta} d \tilde{w}_{1} & \\ d r_{i}=\nabla p_{i-1} d t+\delta \Delta V^{\prime}\left(r_{i}\right) d t-\sqrt{2 \beta^{-1} \delta} \nabla d \tilde{w}_{i-1}, & 2 \leq i \leq N-1 \\ d r_{N}=\nabla p_{N-1} d t+\delta\left(\bar{\tau}(t)+V^{\prime}\left(r_{N-1}\right)-2 V^{\prime}\left(r_{N}\right)\right)-\sqrt{2 \beta^{-1} \delta} \nabla d \tilde{w}_{N-1}, & \\ d p_{1}=\nabla V^{\prime}\left(r_{1}\right) d t+\delta\left(p_{2}-2 p_{1}\right) d t-\sqrt{2 \beta^{-1} \delta} \nabla d w_{1}, & \\ d p_{j}=\nabla V^{\prime}\left(r_{j}\right) d t+\delta \Delta p_{j} d t-\sqrt{2 \beta^{-1} \delta} \nabla d w_{j-1}, & 2 \leq j \leq N-1 \\ d p_{N}=\left(\bar{\tau}(t)-V^{\prime}\left(r_{N}\right)\right) d t-\delta \nabla p_{N-1} d t+\sqrt{2 \beta^{-1} \delta} d w_{N-1} & \end{cases}
$$

Here $\beta^{-1}>0$ is the temperature of the heat bath, and $\left\{w_{i}\right\}_{i=1}^{N-1},\left\{\tilde{w}_{i}\right\}_{i=1}^{N-1}$ are families of independent Brownian motions. The parameter $\delta$ is the intensity of the action of the heat bath, and is chosen depending on $N$ such that $\delta \sim o(N)$. When $\delta=0$, Eq. (1.17) is just the Newton deterministic equations for the Hamiltonian (1.16). Notice the correspondence of the boundary conditions in (1.17) with the one chosen in $(1.12)$.

One of the effects of the action of the stochastic heat bath is to fix, in a large time scale, the variance of the velocities (i.e. the temperature) at $\beta^{-1}$, and establish a local equilibrium, where space-time averages of $V^{\prime}\left(r_{i}\right)$ around a macroscopic particle number $[N x]$ at a macroscopic time $N t$ converges to the equilibrium tension $\tau(r(t, x), \beta)$ at temperature $\beta^{-1}$ and volume stretch $r(t, x)$. Since $\beta$ is fixed by the heat bath and do not evolve in time, we drop it from the notation in the sequel.

The hydrodynamic limit consists in proving that, for any continuous function $G(x)$ on $[0,1]$,

$$
\frac{1}{N} \sum_{i=1}^{N} G\left(\frac{i}{N}\right)\left(\begin{array}{c}
r_{i}(N t) \\
p_{i}(N t)
\end{array}\right) \underset{N \rightarrow \infty}{\longrightarrow} \int_{0}^{1} G(x)\left(\begin{array}{c}
r(t, x) \\
p(t, x)
\end{array}\right) d x,
$$

in probability, with $(r(t, x), p(t, x))$ satisfying (1.5), (1.6). Of course a complete proof would require the uniqueness of such $L^{2}$ valued solutions that satisfy (1.9): this remains an open 
problem. The results contained in [10] states that the limit distribution of the empirical distribution defined on the RHS of (1.18), concentrates on the possible solutions of (1.5) and (1.6) that satisfy (1.9). Since we have no uniqueness result, we cannot assure that the solutions constructed in the present paper coincide with those obtained with the hydrodynamic limit from (1.17). One can however conjecture that this is the case.

This stochastic model was already considered by Fritz [11] in the infinite volume without boundary conditions, and in [9], but without the characterization of the boundary conditions.

In the hydrodynamic limit only $L^{2}$ bounds are available and we are constrained to consider $L^{2}$ valued solutions. Since these solutions do not have definite values on the boundary, boundary conditions have only a dynamical meaning in the sense of an evolution in $L^{2}$ given by (1.5), (1.6).

\section{Hyperbolic system and the existence of weak solutions}

For $r, p: \mathbb{R}_{+} \times[0,1] \rightarrow \mathbb{R}$, consider the hyperbolic system

$$
\left\{\begin{array}{lcc}
r_{t}-p_{x}=0 & p(t, 0)=0 & r(t, 1)=\tau^{-1}(\bar{\tau}(t)) \\
p_{t}-\tau(r)_{x}=0, & p(0, x)=p_{0}(x) & r(0, x)=r_{0}(x)
\end{array}\right.
$$

The nonlinearity $\tau \in C^{3}(\mathbb{R})$ is chosen to have the following properties.

$(\tau-\mathrm{i}) \quad c_{1} \leq \tau^{\prime}(r) \leq c_{2}$ for some $c_{1}, c_{2}>0$ and all $r \in \mathbb{R}$;

$\left(\tau\right.$-ii) $\tau^{\prime \prime}(r) \neq 0$ for all $r \in \mathbb{R}$;

$\left(\tau\right.$-iii) $\tau^{\prime \prime}(r), \tau^{\prime \prime \prime}(r) \in L^{2}(\mathbb{R}) \cap L^{\infty}(\mathbb{R})$.

We also assume that $\bar{\tau}: \mathbb{R}_{+} \rightarrow \mathbb{R}$ is smooth. Moreover, there is a time $T_{\star}$ such that $\bar{\tau}^{\prime}(t)=0$ for all $t \geq T_{\star}$. The initial data $r_{0}, p_{0} \in L^{2}(0,1)$ are compatible with the boundary conditions.

Remark. Conditions $(\tau-\mathrm{i})$ and ( $\tau$-ii) ensure that the system is strictly hyperbolic and genuinely nonlinear, respectively. Condition $(\tau$-iii) is used later on to ensure some boundedness properties of the Lax entropies.

Theorem 2.1. System (2.1) admits a weak solution $\bar{u}=(\bar{r}, \bar{p})$ in the sense of (1.5) and (1.6), such that $\bar{u} \in L^{\infty}\left(0, T ; L^{2}(0,1)\right), \bar{u}(0, x)=u_{0}(x)$ for a.e. $x$; and it satisfies the Clausius inequality:

$$
\mathcal{F}(\bar{u}(t))-\mathcal{F}\left(u_{0}\right) \leq W(t), \quad \forall t \in[0, T]
$$

with $W(t)$ as in (1.10). Furthermore $\bar{u}$ satisfies the local Lax entropy condition in the sense specified in Section 6.

\section{Viscous approximation and energy estimates}

We consider the following parabolic approximation of the hyperbolic system (2.1)

$$
\left\{\begin{array}{l}
r_{t}^{\delta}-p_{x}^{\delta}=\delta r_{x x}^{\delta} \\
p_{t}^{\delta}-\tau\left(r^{\delta}\right)_{x}=\delta p_{x x}^{\delta}
\end{array}, \quad(t, x) \in \mathbb{R}_{+} \times[0,1]\right.
$$

for $\delta>0$, with the boundary conditions: 


$$
p^{\delta}(t, 0)=0, \quad r^{\delta}(t, 1)=\tau^{-1}(\bar{\tau}(t)), \quad p_{x}^{\delta}(t, 1)=0, \quad r_{x}^{\delta}(t, 0)=0,
$$

and initial data:

$$
p^{\delta}(0, x)=p_{0}^{\delta}(x), \quad r^{\delta}(0, x)=r_{0}^{\delta}(x) .
$$

The initial data $r_{0}^{\delta}, p_{0}^{\delta} \in C^{\infty}([0,1])$ are mollifications of $r_{0}$ and $p_{0}$ compatible with the boundary conditions:

$$
p_{0}^{\delta}(0)=0 \quad r_{0}^{\delta}(1)=\tau^{-1}(\bar{\tau}(0)), \quad \partial_{x} p_{0}^{\delta}(1)=0 \quad \partial_{x} r_{0}^{\delta}(0)=0 .
$$

Moreover, there is $C$ independent of $\delta$ such that

$$
\left\|r_{0}^{\delta}\right\|_{L^{2}}+\left\|p_{0}^{\delta}\right\|_{L^{2}}+\left\|\sqrt{\delta} \partial_{x} r_{0}^{\delta}\right\|_{L^{2}} \cdot+\left\|\sqrt{\delta} \partial_{x} p_{0}^{\delta}\right\|_{L^{2}} \leq C
$$

and $\left(r_{0}^{\delta}, p_{0}^{\delta}\right) \rightarrow\left(r_{0}, p_{0}\right)$ strongly in $L^{2}(0,1)$.

As shown in [12] in a more general setting, this system admits a global classical solution $\left(r^{\delta}, p^{\delta}\right)$, with

$$
r^{\delta}, p^{\delta} \in C^{1}\left(\mathbb{R}_{+} ; C^{0}([0,1])\right) \cap C^{0}\left(\mathbb{R}_{+} ; C^{2}([0,1])\right)
$$

Remark. (i) We added two extra Neumann conditions, namely $p_{x}^{\delta}(t, 1)=r_{x}^{\delta}(t, 0)=0$. These conditions reflect the conservative nature of the viscous perturbation, and are required in order to obtain the correct production of free energy.

(ii) One could introduce a nonlinear viscosity term: $\delta \tau\left(r^{\delta}\right)_{x x}$ in place of $\delta r_{x x}^{\delta}$. This is a term which comes naturally from a microscopic derivation of system (3.1), as described in the introduction (see also [13]). Nevertheless, this does not drastically change the problem, thus we shall consider only the linear viscosity $\delta r_{x x}^{\delta}$.

Theorem 3.1 (Energy estimate). There there is a constant $C>0$ independent of $t$ and $\delta$ such that

$$
\int_{0}^{1}\left|u^{\delta}(t, x)\right|^{2} d x+\delta \int_{0}^{t} \int_{0}^{1}\left|u_{x}^{\delta}(s, x)\right|^{2} d x d s \leq C
$$

for all $t \geq 0$ and $\delta>0$.

Proof. Let $F$ be a primitive of $\tau$ such that $\frac{c_{1}}{2} r^{2} \leq F(r) \leq \frac{c_{2}}{2} r^{2}$. By a direct calculation we have

$$
\begin{aligned}
\int_{0}^{1}\left(\frac{\left(p^{\delta}\right)^{2}}{2}+F\left(r^{\delta}\right)\right) & \left.d x\right|_{t=0} ^{t=T}+\int_{0}^{T} \int_{0}^{1}\left(\delta\left(r_{x}^{\delta}\right)^{2}+\delta\left(p_{x}^{\delta}\right)^{2}\right) d x d t=\int_{0}^{T} \bar{\tau}(t) \int_{0}^{1} r_{t}^{\delta} d x d t \\
& =\left.\left(\bar{\tau}(t) \int_{0}^{1} r^{\delta} d x\right)\right|_{t=0} ^{t=T}-\int_{0}^{T} \bar{\tau}^{\prime}(t) \int_{0}^{1} r^{\delta} d x d t
\end{aligned}
$$

Write, for some $\varepsilon>0$ to be chosen later,

$$
\bar{\tau}(T) \int_{0}^{1} r^{\delta}(T, x) d x \leq|\bar{\tau}(T)|\left(\frac{1}{2 \varepsilon}+\frac{\varepsilon}{2} \int_{0}^{1}\left(r^{\delta}\right)^{2}(T, x) d x\right) \leq \frac{C_{\bar{\tau}}}{2 \varepsilon}+\frac{C_{\bar{\tau}} \varepsilon}{2} \int_{0}^{1}\left(r^{\delta}\right)^{2}(T, x) d x
$$

where $C_{\bar{\tau}}=\sup _{t \geq 0}\left(|\bar{\tau}(t)|+\left|\bar{\tau}^{\prime}(t)\right|\right)$ depends on $\bar{\tau}$ only. 
Using $F(r) \geq \frac{c_{1}}{2} r^{2}$ we obtain

$$
\begin{aligned}
\left(\frac{c_{1}}{2}-\frac{C_{\bar{\tau}} \varepsilon}{2}\right) \int_{0}^{1}\left(r^{\delta}\right)^{2}(T, x) d x+\frac{1}{2} \int_{0}^{1}\left(p^{\delta}\right)^{2}(T, x) d x & +\int_{0}^{T} \int_{0}^{1}\left(\delta\left(r_{x}^{\delta}\right)^{2}+\delta\left(p_{x}^{\delta}\right)^{2}\right) d x d t \\
& \leq \frac{C_{\bar{\tau}}}{2 \varepsilon}+C_{0}-\int_{0}^{T} \bar{\tau}^{\prime}(t) \int_{0}^{1} r^{\delta}(t, x) d x d t .
\end{aligned}
$$

Recall that there is $T_{\star}>0$ such that $\bar{\tau}^{\prime}(t)=0$ for $t \geq T_{\star}$. Then, for $T<T_{\star}$, we write

$$
\begin{aligned}
\left(\frac{c_{1}}{2}-\frac{C_{\bar{\tau}} \varepsilon}{2}\right) \int_{0}^{1}\left(r^{\delta}\right)^{2}(T, x) d x & +\frac{1}{2} \int_{0}^{1}\left(p^{\delta}\right)^{2}(T, x) d x+\int_{0}^{T} \int_{0}^{1}\left(\delta\left(r_{x}^{\delta}\right)^{2}+\delta\left(p_{x}^{\delta}\right)^{2}\right) d x d t \\
\leq & \frac{C_{\bar{\tau}}}{2 \varepsilon}+C_{0}+\frac{C_{\bar{\tau}}^{2}}{2} T+\frac{1}{2} \int_{0}^{T} \int_{0}^{1}\left(r^{\delta}\right)^{2}(t, x) d x \\
\leq \frac{C_{\bar{\tau}}}{2 \varepsilon}+C_{0}+\frac{C_{\bar{\tau}}^{2}}{2} T & +\frac{1}{2} \int_{0}^{T} \int_{0}^{1}\left(\left(r^{\delta}\right)^{2}(t, x)+\left(p^{\delta}\right)^{2}(t, x)\right) d x d t \\
& +\frac{1}{2} \int_{0}^{T} \int_{0}^{t} \int_{0}^{1}\left(\delta\left(r_{x}^{\delta}\right)^{2}+\delta\left(p_{x}^{\delta}\right)^{2}\right) d x d s d t
\end{aligned}
$$

where $C_{0}$ depends on the initial data only. Choosing $\varepsilon=c_{1} /\left(2 C_{\bar{\tau}}\right)$ gives

$$
\frac{c_{1}}{4} J(T) \leq C_{0}+\frac{C_{\bar{\tau}}^{2}}{c_{1}}+\frac{C_{\bar{\tau}}^{2}}{2} T+\frac{1}{2} \int_{0}^{T} J(t) d t
$$

where

$$
J(t)=\int_{0}^{1}\left(\left(r^{\delta}\right)^{2}(t, x)+\left(p^{\delta}\right)^{2}(t, x)\right) d x+\int_{0}^{t} \int_{0}^{1}\left(\delta\left(r_{x}^{\delta}\right)^{2}+\delta\left(p_{x}^{\delta}\right)^{2}\right) d x d s .
$$

We apply Gronwall's inequality. This, together with $T<T_{\star}$, gives

$$
\begin{aligned}
J(T) & \leq \frac{4 c_{1} C_{0}+2 C_{\bar{\tau}}^{2}\left(2+c_{1} T\right)}{c_{1}^{2}} \exp \left(\frac{2 T}{c_{1}}\right) \\
& \leq \frac{4 c_{1} C_{0}+2 C_{\bar{\tau}}^{2}\left(2+c_{1} T_{\star}\right)}{c_{1}^{2}} \exp \left(\frac{2 T_{\star}}{c_{1}}\right):=C_{0}\left(c_{1}, \bar{\tau}\right),
\end{aligned}
$$

for all $T \in\left[0, T_{\star}\right)$, where $C_{0}\left(c_{1}, \bar{\tau}\right)$ is independent of $T$ and $\delta$

On the other hand, if $T \geq T_{\star}$, we have

$$
\begin{array}{r}
\left(\frac{c_{1}}{2}-\frac{C_{\bar{\tau}} \varepsilon}{2}\right) \int_{0}^{1}\left(r^{\delta}\right)^{2}(T, x) d x+\frac{1}{2} \int_{0}^{1}\left(p^{\delta}\right)^{2}(T, x) d x+\int_{0}^{T} \int_{0}^{1}\left(\delta\left(r_{x}^{\delta}\right)^{2}+\delta\left(p_{x}^{\delta}\right)^{2}\right) d x d t \\
\leq \frac{C_{\bar{\tau}}}{2 \varepsilon}+C_{0}-\int_{0}^{T_{\star}} \bar{\tau}^{\prime}(t) \int_{0}^{1} r^{\delta}(t, x) d x d t
\end{array}
$$

and the integral at the right-hand side is uniformly bounded in $T, \delta$ and $\delta$, since 


$$
\begin{aligned}
-\int_{0}^{T_{\star}} \bar{\tau}^{\prime}(t) \int_{0}^{1} r^{\delta}(t, x) d x d t & \leq C_{\bar{\tau}} \int_{0}^{T_{\star}} \int_{0}^{1}\left|r^{\delta}(t, x)\right| d x d t \\
& \leq C_{\bar{\tau}} T_{\star}\left(\frac{1}{T_{\star}} \int_{0}^{T_{\star}} \int_{0}^{1}\left(r^{\delta}\right)^{2}(t, x) d x d t\right)^{1 / 2} \\
& \leq C_{\bar{\tau}} \sqrt{T_{\star}}\left(\int_{0}^{T_{\star}} J(t) d t\right)^{1 / 2} \\
& \leq C_{\bar{\tau}} T_{\star} \sqrt{C_{0}\left(c_{1}, \bar{\tau}\right)}
\end{aligned}
$$

From (3.7) we also immediately obtain the following

Corollary 3.2 (Viscous Clausius inequality).

$$
\mathcal{F}\left(u^{\delta}(t)\right)-\mathcal{F}\left(u_{0}^{\delta}\right) \leq-\int_{0}^{t} \bar{\tau}^{\prime}(s) \int_{0}^{1} r^{\delta}(s, x) d x+\bar{\tau}(t) \int_{0}^{1} r^{\delta}(t, x) d x-\bar{\tau}(0) \int_{0}^{1} r_{0}^{\delta}(x) d x
$$

\section{4. $L^{2}$ young measures and compensated compactness}

Throughout this section, for any fixed $T>0$ let $u^{\delta}(t, x):=\left(r^{\delta}(t, x), p^{\delta}(t, x)\right)$ be a strong solution of (3.1) on $Q_{T}$. By Theorem 3.1 and after a time integration over $[0, T]$ we obtain

$$
\left\|u^{\delta}\right\|_{L^{2}\left(Q_{T}\right)} \leq C
$$

for some $C$ independent of $\delta$. Thus we can extract from $\left\{u^{\delta}\right\}_{\delta>0}$ a subsequence that is weakly convergent in $L^{2}\left(Q_{T}\right)$. Namely, up to a subsequence, there exists $\bar{u}=(\bar{r}, \bar{p}) \in$ $L^{2}\left(Q_{T}\right)$ such that

$$
\lim _{\delta \rightarrow 0} \int_{Q_{T}} u^{\delta} \varphi=\int_{Q_{T}} \bar{u} \varphi, \quad \forall \varphi \in L^{2}\left(Q_{T}\right) .
$$

All the limits $\delta \rightarrow 0$ taken below are intended along a chosen subsequence.

In this section, we want to show that for any $\phi \in L^{2}\left(Q_{T}\right)$ we have

$$
\lim _{\delta \rightarrow 0} \int_{Q_{T}} \phi(t, x) \tau\left(r^{\delta}(t, x)\right) d x d t=\int_{Q_{T}} \phi(t, x) \tau(\bar{r}(t, x)) d x d t .
$$

This is done using a $L^{2}$ version of the compensated compactness, which is usually performed in $L^{\infty}$.

From the solution $u^{\delta}(t, x)$, we define the following Young measure on $Q_{T} \times \mathbb{R}^{2}$ :

$$
\nu_{t, x}^{\delta}:=\delta_{u^{\delta}(t, x)},
$$

which is a Dirac mass centered at $u^{\delta}$, i.e.

$$
\int_{Q_{T}} J(t, x) f\left(u^{\delta}(t, x)\right) d x d t=\int_{Q_{T}} \int_{\mathbb{R}^{2}} J(t, x) f(\xi) d \nu_{t, x}^{\delta}(\xi) d x d t
$$

for all measurable $J: Q_{T} \rightarrow \mathbb{R}$ and $f: \mathbb{R}^{2} \rightarrow \mathbb{R}$. 
Since we have $L^{2}$ bounds on $u^{\delta}$, we refer at $\nu_{t, x}^{\delta}$ as a $L^{2}$-Young measure [14]. In particular we have, from (4.1)

$$
\int_{Q_{T}} \int_{\mathbb{R}^{2}}|\xi|^{2} d \nu_{t, x}^{\delta}(\xi) d x d t \leq C
$$

We call $\mathcal{Y}$ the set of Young measures on $Q_{T} \times \mathbb{R}^{2}$ and we make it a metric space by endowing it with the Prohorov's metric. By proposition 4.1 of [15], the set

$$
K_{C}:=\left\{\nu \in \mathcal{Y}: \int_{Q_{T}} \int_{\mathbb{R}^{2}}|\xi|^{2} d \nu_{t, x}(\xi) d x d t \leq C\right\}
$$

is compact in $\mathcal{Y}$. Then, by the fundamental theorem for Young measures ([14], section 2), there exists $\bar{\nu}_{t, x} \in \mathcal{Y}$ so that, up to a subsequence,

$$
\lim _{\delta \rightarrow 0} \int_{Q_{T}} \int_{\mathbb{R}^{2}} J(t, x) f(\xi) d \nu_{t, x}^{\delta}(\xi) d x d t=\int_{Q_{T}} \int_{\mathbb{R}^{2}} J(t, x) f(\xi) d \bar{\nu}_{t, x}(\xi) d x d t
$$

for all continuous and bounded $J: Q_{T} \rightarrow \mathbb{R}$ and $f: \mathbb{R}^{2} \rightarrow \mathbb{R}$. We shall simply write $\nu^{\delta} \rightarrow \bar{\nu}$ in place of (4.7). By a simple adaptation of proposition 4.2 of [15], (4.7) can be extended to a function $f: \mathbb{R}^{2} \rightarrow \mathbb{R}$ such that $f(\xi) /|\xi|^{2} \rightarrow 0$ as $|\xi| \rightarrow+\infty$.

In order to obtain (4.3), we need to prove that the limit Young measure $\bar{\nu}$ is a Dirac mass: $\bar{\nu}_{t, x}=\delta_{\bar{u}(t, x)}$, for some $\bar{u} \in L^{2}\left(Q_{T}\right)$ and for almost every $(t, x) \in Q_{T}$. This is done using the classical argument by Tartar and Murat.

Definition 4.1. A Lax entropy-entropy flux pair for system (2.1) is a couple of differentiable functions $(\eta, q): \mathbb{R}^{2} \rightarrow \mathbb{R}^{2}$ such that

$$
\left\{\begin{array}{l}
\eta_{r}+q_{p}=0 \\
\tau^{\prime}(r) \eta_{p}+q_{r}=0
\end{array}\right.
$$

We show that Tartar's equation holds for any two suitable entropy pairs $(\eta, q)$ and $\left(\eta^{\prime}, q^{\prime}\right)$ to be specified below and almost all $(t, x) \in Q_{T}$ :

$$
\left\langle\eta q^{\prime}-\eta^{\prime} q, \bar{\nu}_{t, x}\right\rangle=\left\langle\eta, \bar{\nu}_{t, x}\right\rangle\left\langle q^{\prime}, \bar{\nu}_{t, x}\right\rangle-\left\langle\eta^{\prime}, \bar{\nu}_{t, x}\right\rangle\left\langle q, \bar{\nu}_{t, x}\right\rangle,
$$

where

$$
\left\langle f, \bar{\nu}_{t, x}\right\rangle:=\int_{\mathbb{R}^{2}} f(\xi) d \bar{\nu}_{t, x}(\xi)
$$

for any measurable $f$. We employ the following argument due to Shearer [7].

Accordingly to Lemma 2 in [7], there exists a family of half-plain supported entropyentropy fluxes $(\eta, q)$ such that $\eta$ and $q$ are bounded together with their first and second derivatives. These are explicitly given as follows. We define $z(r):=\int_{0}^{r} \sqrt{\tau^{\prime}(\rho)} d \rho$ and we define the Riemann coordinates $w_{1}=p+z, w_{2}=p-z$. We also pass from the dependent variables $\eta, q$ to $H, Q$ as follows:

$$
\begin{aligned}
& \eta=\frac{1}{2}\left(\tau^{\prime}\right)^{-1 / 4}(H+Q) \\
& q=\frac{1}{2}\left(\tau^{\prime}\right)^{+1 / 4}(H-Q)
\end{aligned}
$$


so that (4.8) becomes

$$
\left\{\begin{array}{l}
H_{w_{1}}=a Q \\
H_{w_{2}}=-a Q
\end{array}\right.
$$

where

$$
a\left(w_{1}-w_{2}\right)=\frac{\tau^{\prime \prime}\left(r\left(\frac{w_{1}-w_{2}}{2}\right)\right)}{8\left(\tau^{\prime}\left(r\left(\frac{w_{1}-w_{2}}{2}\right)\right)\right)^{3 / 2}} .
$$

Then we fix $\bar{w}_{1}, \bar{w}_{2} \in \mathbb{R}$ and we solve (4.13) with Goursat data given on the lines $w_{1}=$ $\bar{w}_{1}$ and $w_{2}=\bar{w}_{2}$ :

$$
\begin{aligned}
& H\left(\bar{w}_{1}, w_{2}\right)=g\left(w_{2}\right) \\
& Q\left(w_{1}, \bar{w}_{2}\right)=0,
\end{aligned}
$$

where $g$ is continuous and compactly supported. Then one can explicitly solve (4.13) and get

$$
\begin{aligned}
& H\left(w_{1}, w_{2}\right)=g\left(w_{2}\right)+\sum_{n=1}^{\infty}\left(\mathcal{A}^{n} g\right)\left(w_{1}, w_{2}\right) \\
& Q\left(w_{1}, w_{2}\right)=-\int_{\bar{w}_{2}}^{w_{2}} a\left(w_{1}-v\right) H\left(w_{1}, v\right) d v
\end{aligned}
$$

where the operator $\mathcal{A}$ acts on functions $f \in L_{\text {loc }}^{1}\left(\mathbb{R}^{2}\right)$ as follows:

$$
(\mathcal{A} f)\left(w_{1}, w_{2}\right)=-\int_{\bar{w}_{1}}^{w_{1}} \int_{\bar{w}_{2}}^{w_{2}} a\left(v-w_{2}\right) a(v-u) f(v, u) d u d v .
$$

Finally, going back to $\eta$ and $q$ and using our assumptions on $\tau$ it is possible to show ([7], Lemma 2) that $\eta$ and $q$ are bounded, together with their first and second derivatives.

Now we have a suitable family of entropy-entropy flux pair, we use Tartar-Murat Lemma in order to derive Tartar's equation (4.9). We evaluate $(\eta, q)$ along the approximate solutions $u^{\delta}$ and compute the entropy production:

$$
\eta\left(u^{\delta}\right)_{t}+q\left(u^{\delta}\right)_{x}=\delta\left(\eta_{r} r_{x}^{\delta}+\eta_{p} p_{x}^{\delta}\right)_{x}-\delta\left(\eta_{r r}\left(r_{x}^{\delta}\right)^{2}+\eta_{p p}\left(p_{x}^{\delta}\right)^{2}+2 \eta_{r p} r_{x}^{\delta} p_{x}^{\delta}\right)
$$

Since $\eta_{r}$ and $\eta_{p}$ are bounded and $\sqrt{\delta} r_{x}^{\delta}, \sqrt{\delta} p_{x}^{\delta}$ are bounded in $L^{2}\left(Q_{T}\right)$, we have

$$
\lim _{\delta \rightarrow 0} \delta\left(\eta_{r} r_{x}^{\delta}+\eta_{p} p_{x}^{\delta}\right)_{x}=0 \quad \text { in } H^{-1}\left(Q_{T}\right)
$$

while

$$
\left\|\delta\left(\eta_{r r}\left(r_{x}^{\delta}\right)^{2}+\eta_{p p}\left(p_{x}^{\delta}\right)^{2}+2 \eta_{r p} r_{x}^{\delta} p_{x}^{\delta}\right)\right\|_{L^{1}\left(Q_{T}\right)} \leq C
$$

uniformly with respect to $\delta$. Thus we have obtained an equality of the form

$$
\eta\left(u^{\delta}\right)_{t}+q\left(u^{\delta}\right)_{x}=\chi^{\delta}+\psi^{\delta}
$$


where $\left\{\chi^{\delta}\right\}_{\delta>0}$ lies in a compact set of $H^{-1}\left(Q_{T}\right)$ and $\left\{\psi^{\delta}\right\}_{\delta>0}$ is bounded in $L^{1}\left(Q_{T}\right)$. Moreover, since $\eta$ and $q$ are bounded, $\left\{\eta\left(u^{\delta}\right)_{t}+q\left(u^{\delta}\right)_{x}\right\}_{\delta>0}$ is bounded in $W^{-1, p}\left(Q_{T}\right)$ for some $p>2$.

Therefore, we can apply Tartar-Murat and the div-curl lemma (cf [16], Theorem 16.2.1 and Lemma 16.2.2) and obtain Tartar's equation (4.9).

The final step is to use Tartar's equation to prove that the support of the limit Young measure $\bar{\nu}_{t, x}$ is a point. This is done in lemmas 4 to 7 of [7] and leads to the following

Proposition 4.2. There exists a $\bar{u} \in L^{2}\left(Q_{T}\right)$ such that $\bar{\nu}_{t, x}=\delta_{\bar{u}(t, x)}$ for almost all $(t, x) \in Q_{T}$. Moreover, $u^{\delta} \rightarrow \bar{u}$ strongly in $L^{p}\left(Q_{T}\right)$ for any $p \in[1,2)$.

\subsection{Regularity}

Proposition 4.3. For the function $\bar{u}$ obtained in section 4 ,

$$
\bar{u} \in L^{\infty}\left(0, T ; L^{2}(0,1)\right) .
$$

Proof. Since $u^{\delta} \rightarrow \bar{u}$ in $L^{p}$ strong for $p<2$, we can extract a subsequence $\left\{u^{\delta_{k}}\right\}_{k \in \mathbb{N}}$ that converges pointwise to $\bar{u}$ for almost all $t$ and $x$. In particular, for almost all $t$, the sequence $u^{\delta_{k}}(t, x)$ converges for almost all $x$. Therefore, by Fatou lemma and Theorem 3.1 ,

$$
\int_{0}^{1}|\bar{u}(t, x)|^{2} d x \leq \liminf _{k \rightarrow \infty} \int_{0}^{1}\left|u^{\delta_{k}}(t, x)\right|^{2} d x \leq C
$$

for almost all $t \in[0, T]$.

The proof is of next lemma is standard and therefore omitted.

Lemma 4.4. Let $a(t):=\tau^{-1}(\bar{\tau}(t))$. Then, the solutions $\left(r^{\delta}, p^{\delta}\right)$ of the viscous system (3.1) can be written as follows:

$$
\begin{gathered}
r^{\delta}(t, x)=a(t)+\int_{0}^{1} G_{r}^{\delta}\left(x, x^{\prime}, t\right)\left(r_{0}^{\delta}\left(x^{\prime}\right)-a(0)\right) d x^{\prime} \\
+\int_{0}^{t} \int_{0}^{1} G_{r}^{\delta}\left(x, x^{\prime}, t-t^{\prime}\right)\left(\partial_{x^{\prime}} p^{\delta}\left(t^{\prime}, x^{\prime}\right)-a\left(t^{\prime}\right)\right) d x^{\prime} d t^{\prime} \\
p^{\delta}(t, x)=\int_{0}^{1} G_{p}^{\delta}\left(x, x^{\prime}, t\right) p_{0}^{\delta}\left(x^{\prime}\right) d x^{\prime}+\int_{0}^{t} \int_{0}^{1} G_{p}^{\delta}\left(x, x^{\prime}, t-t^{\prime}\right) \partial_{x^{\prime}} \tau\left(r^{\delta}\left(t^{\prime}, x^{\prime}\right)\right) d x^{\prime} d t^{\prime}
\end{gathered}
$$

where the $G_{r}^{\delta}$ and $G_{p}^{\delta}$ are Green functions of the heat operator $\partial_{t}-\delta \partial_{x x}$ with homogeneous boundary conditions:

$$
\begin{aligned}
& G_{r}^{\delta}\left(1, x^{\prime}, t\right)=\partial_{x} G_{r}^{\delta}\left(0, x^{\prime}, t\right)=0 \\
& G_{p}^{\delta}\left(0, x^{\prime}, t\right)=\partial_{x} G_{p}^{\delta}\left(1, x^{\prime}, t\right)=0
\end{aligned}
$$

for all $x^{\prime} \in[0,1], t \geq 0$ and $\delta>0$. 
The Green's functions $G_{r}^{\delta}\left(x, x^{\prime}, t\right)$ and $G_{p}^{\delta}\left(x, x^{\prime}, t\right)$ are symmetric under the exchange of $x$ and $x^{\prime}$. Moreover we have the following identities

$$
\begin{aligned}
& \partial_{x} G_{p}^{\delta}\left(x, x^{\prime}, t\right)=-\partial_{x^{\prime}} G_{r}^{\delta}\left(x, x^{\prime}, t\right), \\
& \partial_{x} G_{r}^{\delta}\left(x, x^{\prime}, t\right)=-\partial_{x^{\prime}} G_{p}^{\delta}\left(x, x^{\prime}, t\right) .
\end{aligned}
$$

Remark. The functions $G_{r}^{\delta}$ and $G_{p}^{\delta}$ have the following explicit forms:

$$
\begin{aligned}
G_{p}^{\delta}\left(x, x^{\prime}, t\right) & =\frac{1}{2} \sum_{n \text { odd }} e^{-t \delta \lambda_{n}} \sin \left(\sqrt{\lambda_{n}} x\right) \sin \left(\sqrt{\lambda_{n}} x^{\prime}\right) \\
G_{r}^{\delta}\left(x, x^{\prime}, t\right) & =\frac{1}{2} \sum_{n \text { odd }} e^{-t \delta \lambda_{n}} \cos \left(\sqrt{\lambda_{n}} x\right) \cos \left(\sqrt{\lambda_{n}} x^{\prime}\right),
\end{aligned}
$$

with $\lambda_{n}=\frac{n^{2} \pi^{2}}{4}$.

Proposition 4.5. For any $\phi \in C^{1}([0,1])$, the application

$$
t \mapsto I_{\phi}(t):=\int_{0}^{1} \phi(x) \bar{u}(t, x) d x
$$

is Lipschitz continuous. Consequently $\bar{u}(t, \cdot) \in L^{2}(0,1)$ for all $t \geq 0$.

Proof. We prove the statement for $\bar{p}$, as the proof for $\bar{r}$ is similar. Furthermore, we prove the proposition only between 0 and $t$, as in the general case, say between $t_{1}$ and $t$, it is enough to replace the initial term $p_{0}^{\delta}(x)$ with $p^{\delta}\left(t_{1}, x\right)$. We let

$$
I_{\phi}^{\delta}(t):=\int_{0}^{1} \phi(x) p^{\delta}(t, x) d x
$$

and evaluate

$$
\begin{gathered}
I_{\phi}(t)-I_{\phi}(0)=\int_{0}^{1} \int_{0}^{1} \phi(x) G_{p}^{\delta}\left(x, x^{\prime}, t\right) p_{0}^{\delta}\left(x^{\prime}\right) d x^{\prime} d x-\int_{0}^{1} \phi(x) p_{0}^{\delta}(x) d x \\
\quad+\int_{0}^{t} \int_{0}^{1} \int_{0}^{1} \phi(x) G_{p}^{\delta}\left(x, x^{\prime}, t-t^{\prime}\right) \partial_{x^{\prime}} \tau\left(r^{\delta}\left(t^{\prime}, x^{\prime}\right)\right) d x d x^{\prime} d t^{\prime} \\
=\int_{0}^{1} \int_{0}^{1} \phi(x)\left[G_{p}^{\delta}\left(x, x^{\prime}, t\right)-\delta\left(x, x^{\prime}\right)\right] p_{0}^{\delta}\left(x^{\prime}\right) d x^{\prime} d x \\
+\int_{0}^{t} \int_{0}^{1} \int_{0}^{1} \phi(x) \partial_{x} G_{r}^{\delta}\left(x, x^{\prime}, t-t^{\prime}\right) \tau\left(r^{\delta}\left(t^{\prime}, x^{\prime}\right)\right) d x d x^{\prime} d t^{\prime}+\int_{0}^{t} \int_{0}^{1} \phi(x) G_{p}^{\delta}\left(x, 1, t-t^{\prime}\right) \bar{\tau}\left(t^{\prime}\right) d x d t^{\prime},
\end{gathered}
$$

where we have used the symmetry of $G_{p}^{\delta}$ as well as the property $\partial_{x^{\prime}} G_{p}^{\delta}=-\partial_{x} G_{r}^{\delta}$. The boundary term is estimated as

$$
\begin{aligned}
\left|\int_{0}^{t} \int_{0}^{1} \phi(x) G_{p}^{\delta}\left(x, 1, t-t^{\prime}\right) \bar{\tau}\left(t^{\prime}\right) d x d t^{\prime}\right| & \leq\|\bar{\tau}\|_{\infty} \int_{0}^{t}\left|\int_{0}^{1} \phi(x) G_{p}^{\delta}\left(x, 1, t-t^{\prime}\right) d x\right| d t^{\prime} \\
& \leq t\|\bar{\tau}\|_{\infty}\|\phi\|_{L^{2}}
\end{aligned}
$$


We estimate the term involving $\tau$ as

$$
\begin{aligned}
& \left|\int_{0}^{t} \int_{0}^{1} \int_{0}^{1} \phi(x) \partial_{x} G_{r}^{\delta}\left(x, x^{\prime}, t-t^{\prime}\right) \tau\left(r^{\delta}\left(t^{\prime}, x^{\prime}\right)\right) d x d x^{\prime} d t^{\prime}\right| \\
& =\left|\frac{1}{2} \sum_{n \text { odd }} \int_{0}^{1} \sqrt{\lambda_{n}} \sin \left(\sqrt{\lambda_{n}} x\right) \phi(x) d x \int_{0}^{t} d t^{\prime} e^{-\left(t-t^{\prime}\right) \lambda_{n} \delta} \int_{0}^{1} \cos \left(\sqrt{\lambda_{n}} x^{\prime}\right) \tau\left(r^{\delta}\left(t^{\prime}, x^{\prime}\right)\right) d x^{\prime}\right| \\
& \leq\left\|\phi^{\prime}\right\|_{L^{2}}\left[\frac{1}{2} \sum_{n \text { odd }}\left(\int_{0}^{t} d t^{\prime} e^{-\left(t-t^{\prime}\right) \lambda_{n} \delta} \int_{0}^{1} \cos \left(\sqrt{\lambda_{n}} x^{\prime}\right) \tau\left(r^{\delta}\left(t^{\prime}, x^{\prime}\right)\right) d x^{\prime}\right)^{2}\right]^{1 / 2} \\
& \quad \leq\left\|\phi^{\prime}\right\|_{L^{2}}\left[\frac{1}{2} \sum_{n \text { odd }}\left(\frac{1-e^{-2 t \lambda_{n} \delta}}{2 \lambda_{n} \delta}\right) \int_{0}^{t} d t^{\prime}\left(\int_{0}^{1} \cos \left(\sqrt{\lambda_{n}} x^{\prime}\right) \tau\left(r^{\delta}\left(t^{\prime}, x^{\prime}\right)\right) d x^{\prime}\right)^{2}\right]^{1 / 2} \\
& \quad \leq\left\|\phi^{\prime}\right\|_{L^{2}}\left[\frac{t}{2} \int_{0}^{t} d t^{\prime} \sum_{n \text { odd }}\left(\int_{0}^{1} \cos \left(\sqrt{\lambda_{n}} x^{\prime}\right) \tau\left(r^{\delta}\left(t^{\prime}, x^{\prime}\right)\right) d x^{\prime}\right)^{1 / 2}\right]^{1 / 2} \\
& =\left\|\phi^{\prime}\right\|_{L^{2}}\left[t \int_{0}^{t} d t^{\prime} \int_{0}^{1} \tau\left(r^{\delta}\left(t^{\prime}, x^{\prime}\right)\right)^{2} d x^{\prime}\right]^{1 / 2} \\
& \leq t\left\|\phi^{\prime}\right\|_{L^{2}}\left\|\int_{0}^{1} \tau\left(r^{\delta}\left(\cdot, x^{\prime}\right)\right)^{2} d x^{\prime}\right\|_{L^{\infty}(0, T)}^{1 / 2} \leq t \phi^{\prime} \|_{L^{2}}
\end{aligned}
$$

where $C$ is independent of $t$ and $\delta$.

In order to estimate the first term of (4.33) we write

$$
\begin{aligned}
& \int_{0}^{1} \int_{0}^{1} \phi(x)\left[G_{p}^{\delta}\left(x, x^{\prime}, t\right)-\delta\left(x, x^{\prime}\right)\right] p_{0}^{\delta}\left(x^{\prime}\right) d x^{\prime} d x \\
& =\frac{1}{2} \sum_{n \text { odd }} \int_{0}^{1} \sin \left(\sqrt{\lambda_{n}} x\right) \phi(x) d x\left(e^{-t \lambda_{n} \delta}-1\right) \int_{0}^{1} \sin \left(\sqrt{\lambda_{n}} x^{\prime}\right) p_{0}^{\delta}\left(x^{\prime}\right) d x^{\prime} \\
& \leq t \delta \frac{1}{2} \sum_{n \text { odd }} \lambda_{n}\left|\int_{0}^{1} \sin \left(\sqrt{\lambda_{n}} x\right) \phi(x) d x \| \int_{0}^{1} \sin \left(\sqrt{\lambda_{n}} x^{\prime}\right) p_{0}^{\delta}\left(x^{\prime}\right) d x^{\prime}\right| \\
& \leq t\left\|\phi^{\prime}\right\|_{L^{2}}\left\|\delta \partial_{x} p_{0}^{\delta}\right\|_{L^{2}} \leq t\left\|\phi^{\prime}\right\|_{L^{2}} C
\end{aligned}
$$

where we have used the assumption that $\left\{\sqrt{\delta} \partial_{x} p_{0}^{\delta}\right\}_{\delta>0}$ is bounded in $L^{2}(0,1)$. We have also assumed, without loss of generality, $\delta \leq 1$.

Putting everything together, we have obtained

$$
\left|I_{\phi}^{\delta}(t)-I_{\phi}^{\delta}(0)\right| \leq t C\left(\left\|\phi^{\prime}\right\|_{L^{2}}+\|\phi\|_{L^{2}}\right)
$$

for some constant $C$ independent of $t$ and $\delta$. This leads to the conclusion after passing to the limit $\delta \rightarrow 0$. 


\section{Proof of Theorem 2.1 and Clausius inequality}

All is left to prove is that the function $\bar{u}$ obtained in the previous section is to a weak solution of the hyperbolic system (2.1), in the sense of Section 2 . Let $\psi \in C^{1}\left(Q_{T}\right)$ with $\psi(t, 0)=0$ for all $t \in[0, T]$. Then, for any $t \in[0, T]$ we have

$$
\begin{aligned}
0= & \int_{0}^{t} \int_{0}^{1}\left(\psi p_{s}^{\delta}-\psi \tau\left(r^{\delta}\right)_{x}-\delta \psi p_{x x}^{\delta}\right) d x d s \\
= & \int_{0}^{1} \psi(t, x) p^{\delta}(t, x) d x-\int_{0}^{1} \psi(0, x) p_{0}^{\delta}(x) d x+ \\
& -\int_{0}^{t} \int_{0}^{1}\left(\psi_{s} p^{\delta}-\psi_{x} \tau\left(r^{\delta}\right)-\delta \psi_{x} p_{x}^{\delta}\right) d x d s-\int_{0}^{\infty} \psi(t, 1) \bar{\tau}(t) d t
\end{aligned}
$$

where we have used the initial-boundary conditions $\tau\left(r^{\delta}(t, 1)\right)=\bar{\tau}(t)$ and $p_{x}^{\delta}(t, 1)=$ $0, p^{\delta}(0, x)=p_{0}^{\delta}(x)$ as well as $\psi(t, 0)=0$. Since $p_{0}^{\delta}$ converges to $p_{0}$ in $L^{2}(0,1)$, we have

$$
\lim _{\delta \rightarrow 0} \int_{0}^{1} \psi(0, x) p_{0}^{\delta}(x) d x=\int_{0}^{1} \psi(0, x) p_{0}(x) d x .
$$

Furthermore, Theorem 3.1 implies $\sqrt{\delta} p_{x}^{\delta} \in L^{2}\left(Q_{T}\right)$, consequently $\int_{0}^{t} \int_{0}^{1} \delta \psi_{x} p_{x}^{\delta} d x d s$ vanishes as $\delta \rightarrow 0$. Moreover, (4.2) implies, along the subsequence that defines $\bar{u}=(\bar{r}, \bar{p})$,

$$
\lim _{\delta \rightarrow 0} \int_{0}^{t} \int_{0}^{1} \psi_{t} p^{\delta} d x d s=\int_{0}^{t} \int_{0}^{1} \psi_{s} \bar{p} d x d t
$$

while by (4.3) we have that

$$
\lim _{\delta \rightarrow 0} \int_{0}^{t} \int_{0}^{1} \psi_{x} \tau\left(r^{\delta}\right) d x d s=\int_{0}^{t} \int_{0}^{1} \psi_{x} \tau(\bar{r}) d x d s,
$$

so that (1.6) is satisfied. The (1.5) is linear and it follows similarly.

Proposition 5.1. The solution $\bar{u}$ satisfies Clausius inequality

$$
\mathcal{F}(\bar{u}(t))-\mathcal{F}\left(u_{0}\right) \leq W(t)
$$

for all $t \in[0, T]$, where

$$
W(t)=-\int_{0}^{t} \bar{\tau}^{\prime}(s) \int_{0}^{1} \bar{r}(s, x) d x+\bar{\tau}(t) \int_{0}^{1} \bar{r}(t, x) d x-\bar{\tau}(0) \int_{0}^{1} r_{0}(x) d x .
$$

Proof. By Proposition 4.3, Corollary 3.1, and Lemma 4.5, we have, for all $t \in[0, T]$,

$$
\begin{aligned}
& \int_{0}^{1}\left(\frac{\bar{p}^{2}(t, x)}{2}+F(\bar{r}(t, x))\right) d x \leq \liminf _{k \rightarrow \infty} \int_{0}^{1}\left(\frac{\left(p^{\delta_{k}}\right)^{2}(t, x)}{2}+F\left(r^{\delta_{k}}(t, x)\right)\right) d x \\
& \leq \lim _{k \rightarrow \infty}\left(\mathcal{F}\left(u_{0}^{\delta_{k}}\right)-\int_{0}^{t} \bar{\tau}^{\prime}(s) \int_{0}^{1} r^{\delta_{k}}(s, x) d x d s+\bar{\tau}(t) \int_{0}^{1} r^{\delta_{k}}(t, x) d x-\bar{\tau}(0) \int_{0}^{1} r_{0}^{\delta_{k}}(x) d x\right) \\
&=\mathcal{F}\left(u_{0}\right)-\int_{0}^{t} \bar{\tau}^{\prime}(s) \int_{0}^{1} \bar{r}(s, x) d x d s+\bar{\tau}(t) \int_{0}^{1} \bar{r}(t, x) d x-\bar{\tau}(0) \int_{0}^{1} r_{0}(x) d x
\end{aligned}
$$


where we have used the fact that $u_{0}^{\delta}$ converges to $u_{0}$ in $L^{2}$ strongly in order to conclude that $\mathcal{F}\left(u_{0}^{\delta}\right) \rightarrow \mathcal{F}\left(u_{0}\right)$. Moreover, all the integrals are well defined, since the application

$$
t \mapsto \int_{0}^{1} \bar{r}(t, x) d x
$$

is continuous.

Thanks to the Clausius inequality, the solutions we have constructed are natural candidates for being the thermodynamic entropy solution of Eq. (3.1) and one can conjecture that such limit is unique.

\section{Lax entropy condition}

From (4.18), if $\eta$ is a convex Lax entropy, we have that

$$
\eta\left(u^{\delta}\right)_{t}+q\left(u^{\delta}\right)_{x} \geq \delta\left(\eta_{r} r_{x}^{\delta}+\eta_{p} p_{x}^{\delta}\right)_{x} .
$$

If $\eta$ grows at most quadratically, the right hand side of (6.1) vanished in $H^{-1}\left(Q_{T}\right)$, and by (4.19), for the limit we have that $\eta(u)_{t}+q(u)_{x} \geq 0$ as a distribution in $H^{-1}\left(Q_{T}\right)$. This is the usual local characterization of weak entropy solutions, that is independent of the boundary conditions and does not give informations of the behavior at the boundary. Our solutions obtained from viscosity approximation satisfy such local entropy condition.

Our point is that this local characterization should be implemented by the global entropy production for the entropy given by the free energy $\eta(u)=\mathcal{F}(u)$, i.e. the Clausius inequality.

\section{Acknowledgments}

We thank Olivier Glass for very helpful discussions. We also thank an anonymous referee, whose critics and comments helped us to improve the first version of this article.

\section{Funding}

This work has been partially supported by the grants ANR-15-CE40-0020-01 LSD of the French National Research Agency.

\section{ORCID}

Stefano Marchesani (D) http://orcid.org/0000-0002-0471-2318

Stefano Olla (D) http://orcid.org/0000-0002-0471-2318

\section{References}

[1] Chen, G.-Q., Frid, H. (1999). Vanishing viscosity limit for initial-boundary value problems for conservation laws. Contemp. Math. 238:35-51.

[2] Otto, F. (1996). Initial-boundary value problem for a scalar conservation law. C. R. Acad. Sci. Sér. Math. 322:729-734. 
[3] Evans, L. (2004). A survey of entropy methods for partial differential equations. Bull. Amer. Math. Soc. 41(4):409-438. DOI: 10.1090/S0273-0979-04-01032-8.

[4] Ball, J. M., Chen, G.-Q. G. (2013). Entropy and convexity for nonlinear partial differential equations. arXiv preprint arXiv:1310.7975.

[5] Serre, D. (1986). La compacité par compensation pour les systèmes hyperboliques non linéaires de deux équations á une dimension d'espace. J. Math. Pures Appl. 65(4):423-468.

[6] Serre, D., Shearer, J. (1994). Convergence with physical viscosity for nonlinear elasticity. Preprint, unpublished.

[7] Shearer, J. W. (1994). Global existence and compactness in $L^{p}$ for the quasi-linear wave equation. Commun. Partial Differ. Equ. 19(11):1829-1878.

[8] Lin, P. (1992). Young measures and an application of compensated compactness to onedimensional nonlinear elastodynamics. Trans. Amer. Math. Soc. 329(1):377-413. DOI: 10. 1090/S0002-9947-1992-1049615-0.

[9] Marchesani, S., Olla, S. (2018). Hydrodynamic limit for an anharmonic chain under boundary tension. Nonlinearity. 31(11):4979-5035. DOI: 10.1088/1361-6544/aad675.

[10] Marchesani, S., Olla, S. (2020). Hydrodynamic limits and Clausius inequality for isothermal non-linear elastodynamics with boundary tension. arXiv preprint arXiv:1911.13167.

[11] Fritz, J. (2011). Microscopic theory of isothermal elastodynamics. Arch. Ration. Mech. Anal. 201(1):209-249. DOI: 10.1007/s00205-010-0385-8.

[12] Alasio, L., Marchesani, S. (2019). Global existence for a class of viscous systems of conservation laws. Nonlinear Differ. Equ. Appl. 26(5):32. DOI: 10.1007/s00030-019-0577-3.

[13] Marchesani, S. (2020). Hydrodynamic limit for a diffusive system with boundary conditions. arXiv preprint arXiv:1903.08576.

[14] Ball, J. M. (1989). A version of the fundamental theorem for young measures. In: Rascle M., Serre D., Slemrod M., eds. PDEs and Continuum Models of Phase Transitions. Vol. 344. Lecture Notes in Physics, Berlin/Heidelberg: Springer, pp. 207-215.

[15] Berthelin, F., Vovelle, J. (2017). Stochastic isentropic Euler equations. Anna. ENS (to appear).

[16] Dafermos, C. M. (2010). Hyperbolic Conservation Laws in Continuum Physics. Grundlehren Der Mathematischen Wissenschaften [Fundamental Principles of Mathematical Sciences], Vol. 325, Springer Science \& Business Media. 